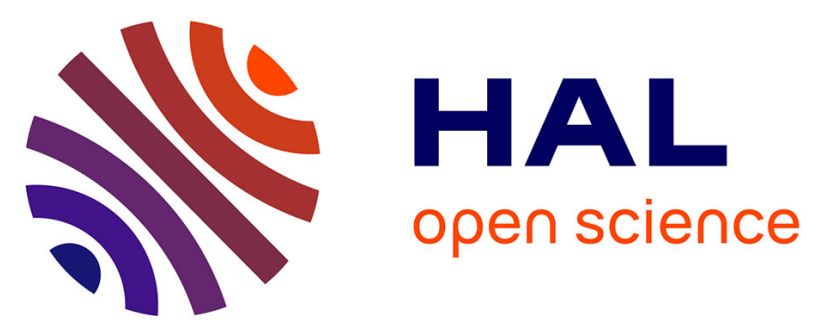

\title{
Twenty Years of Coordination Technologies: State-of-the-Art and Perspectives
}

Giovanni Ciatto, Stefano Mariani, Maxime Louvel, Andrea Omicini, Franco Zambonelli

\section{- To cite this version:}

Giovanni Ciatto, Stefano Mariani, Maxime Louvel, Andrea Omicini, Franco Zambonelli. Twenty Years of Coordination Technologies: State-of-the-Art and Perspectives. 20th International Conference on Coordination Languages and Models (COORDINATION), Jun 2018, Madrid, Spain. pp.51-80, 10.1007/978-3-319-92408-3_3. hal-01821495

\section{HAL Id: hal-01821495 \\ https://hal.inria.fr/hal-01821495}

Submitted on 22 Jun 2018

HAL is a multi-disciplinary open access archive for the deposit and dissemination of scientific research documents, whether they are published or not. The documents may come from teaching and research institutions in France or abroad, or from public or private research centers.
L'archive ouverte pluridisciplinaire HAL, est destinée au dépôt et à la diffusion de documents scientifiques de niveau recherche, publiés ou non, émanant des établissements d'enseignement et de recherche français ou étrangers, des laboratoires publics ou privés. 


\title{
Twenty Years of Coordination Technologies: State-of-the-Art and Perspectives
}

\author{
Giovanni Ciatto[0000-0002-1841-8996]†, Stefano Mariani ${ }^{[0000-0001-8921-8150] \star \text {, }}$ \\ Maxime Louve $\left[^{[0000-0002-1330-253 X] \diamond}\right.$, Andrea Omicini ${ }^{[0000-0002-6655-3869] \dagger}$, \\ and Franco Zambonelli ${ }^{[0000-0002-6837-8806] \star ~}$ \\ $\dagger$ Alma Mater Studiorum-Università di Bologna, Italy \\ giovanni.ciatto@unibo.it, andrea.omicini@unibo.it \\ * Università di Modena e Reggio Emilia, Italy \\ stefano.mariani@unimore.it, franco.zambonelli@unimore.it \\ $\diamond$ Bag-Era, France \\ maxime.louvel@bag-era.fr
}

\begin{abstract}
Since complexity of inter- and intra-systems interactions is steadily increasing in modern application scenarios (e.g., the IoT), coordination technologies are required to take a crucial step towards maturity. In this paper we look back at the history of the COORDINATION conference in order to shed light on the current status of the coordination technologies there proposed throughout the years, in an attempt to understand success stories, limitations, and possibly reveal the gap between actual technologies, theoretical models, and novel application needs.
\end{abstract}

Keywords: coordination technologies, middleware, survey

\section{Scope, Goal, and Method}

Complexity of computational systems, as well as their impact on our everyday life, is constantly increasing, along with the growing complexity of interactioninter- and intra-systems. Accordingly, the role of coordination models should expectedly grow, along with the relevance of coordination technologies within ICT systems: instead, this is apparently not happening-yet.

Then, it is probably the right time - now, after twenty years of the COORDINATION conference - and the right place - the COORDINATION conference itself - to take a a step back and reflect on what happened to coordination models, languages, and (above all) technologies in the last two decades. That is why in this paper we survey all the technologies presented and discussed at COORDINATION, examine their stories and their current status, and try to provide an overall view of the state-of-the art of coordination technologies as emerging from twenty years of work by the COORDINATION community. The main goal is to provide a sound basis to answer questions such as: Are coordination technologies ready for the industry? If not, what is currently missing? Which archetypal models lie behind them? Which are the research areas most/least explored? And what about the target application scenarios? 


\subsection{Structure \& Contribution of the Paper}

Section 2 first provides an overview of the data about papers published in the conference throughout the years (Subsection 2.1), as collected from the official SpringerLink website and its companion BookMetrix service, with the aim of emphasising trends concerning (i) the number of papers published in each volume, (ii) the number of citations generated by each volume, (iii) the number of downloads generated by each volume, (iv) the most cited paper of each volume, and $(v)$ the most downloaded paper of each volume.

Then, the scope of our analysis narrows down to those papers bringing a technological contribution, in the sense of describing a software artefact offering an API exploitable by other software to coordinate its components. Accordingly, Subsection 2.2 provides an overview of all the technologies born within the COORDINATION conference series. For each one, the reference model implemented, and the web URL where to retrieve the software - if any - are given.

Then, a brief description of all the software for which no working implementation could be found is reported for the sake of completeness, whereas technologies still available are thoroughly described in Subsection 2.3. There, each selected technology was downloaded and tested to clearly depict its health status:

- date of last update to the source code (or project web page, if the former is not available)

- whether the software appears to be actively developed, in maintenance mode, or discontinued

- whether suitable documentation is available

- whether the source code is publicly available

- whether the build process of the software artefact is reproducible

- whether the software artefact, once built, executes with no errors

For the latter two items, in case of failures, an explanation of the problem and, if needed, the steps undertaken in the attempt to overcome it, are provided too. In particular, the latter test is not meant to measure performance, or, to provide a benchmark for comparisons: its purpose is to assess whether the technology is usable, that is, executable on nowadays software platforms and by nowadays programming languages. For instance, a library requiring an obsolete third-party libraries that hinders smooth deployment is considered not usable. Accordingly, each technology is tested either running provided example code, or developing a minimal working example of usage of the API.

Section 3 discusses the data collected so as to deliver insights about: (i) the evolution of technologies as they are stemming from a few archetypal models (Fig. 5), (ii) the relationships between the selected technologies, as a comparison of their features (Fig. 6), and (iii) the main goal and reference scenario of each technology (Fig. 7). Also, a general discussion is provided, reporting about success stories, peculiarities, and opportunities.

Finally, Section 4 concludes the paper by summarising the results of the survey and providing some perspectives for the future of coordination technologies. 


\subsection{Method}

The scope of this survey is indeed the COORDINATION conference series. There, we focus on coordination technologies intended as software implementing a given coordination model, language, mechanism, or approach with the goal of providing coordination services to other software applications. In other words, our focus is on technologies implementing some form of coordination middleware or API-analysed in Subsection 2.2. We nevertheless include in our overview other technologies produced within COORDINATION (Subsection 2.1), such as simulation frameworks, model-checking tools, and proof-of-concept implementations of process algebras - which are only described in short.

Starting from the COORDINATION conference proceedings available online from SpringerLink 1 , the survey proceeds as follows:

1. for each conference year, papers describing a coordination-related technology were gathered manually into a Google Spreadsheet

2. for each collected paper, we checked whether the paper was actually proposing some software package - papers failing the test are omitted

3. for each paper passing the test, we verified the health status of the technologyas described in Subsection 1.1

4. then, for each paper featuring at least a usable distribution - meaning a downloadable version of the software - the corresponding software was downloaded and tested-i.e., installation \& basic usage

\section{The Survey}

Although the focus of this paper are coordination technologies, we believe an overview of the whole conference proceedings is due to give context to the survey itself. Accordingly, Subsection 2.1 summarises and analyses all the data officially available from Springer-concerning, for instance, citations and downloads of each volume and paper. Then, Subsection 2.2 accounts for all the coordination technologies mentioned in COORDINATION papers, regardless of their actual availability, while Subsection 2.3 reports about the core of this survey: the status of the coordination technologies nowadays publicly available.

\subsection{Overview}

The COORDINATION conference series has been held 19 times since its first edition in 1996 in Cesena (Italy), and generated as many conference proceedings volumes - all available online ${ }^{1}$. Data about the number of published papers, the number of citations and downloads per year of each volume, as well as the most cited and most download paper have been collected from SpringerLink and its companion service BookMetrix ${ }^{2}$ and are reported in Table 1 on page 4 (last checked February 9th, 2018). Highest values for each column are emphasised in bold. 


\begin{tabular}{|c|c|c|c|c|c|}
\hline Edition & No. of papers & Citations/Year & Downloads/Year & MCP & MDP \\
\hline 1996 & 34 & 3.32 & 140.00 & 16 & 124 \\
\hline 1997 & 31 & 2.86 & 140.48 & 14 & 149 \\
\hline 1999 & 32 & 2.05 & 205.26 & 6 & 154 \\
\hline 2000 & 27 & 0.11 & - & 6 & 158 \\
\hline 2002 & 35 & 2.81 & 301.88 & 7 & 180 \\
\hline 2004 & 23 & 4.07 & 197.86 & 19 & 146 \\
\hline 2005 & 19 & 3.00 & 261.54 & 9 & 214 \\
\hline 2006 & 18 & 6.25 & 312.50 & 22 & 297 \\
\hline 2007 & 17 & 8.27 & 341.82 & 14 & 308 \\
\hline 2008 & 21 & 10.70 & 391.00 & 13 & 227 \\
\hline 2009 & 15 & 7.44 & 370.00 & 13 & 259 \\
\hline 2010 & 12 & 3.25 & 507.50 & 6 & 536 \\
\hline 2011 & 14 & 4.00 & 538.57 & 6 & 675 \\
\hline 2012 & 18 & 5.50 & 1081.67 & 6 & 523 \\
\hline 2013 & 17 & 8.20 & 1314.00 & 7 & 547 \\
\hline 2014 & 12 & 10.50 & 792.50 & 10 & 299 \\
\hline 2015 & 15 & 6.67 & 1453.33 & 11 & 336 \\
\hline 2016 & 16 & 14.00 & 2355.00 & 4 & 350 \\
\hline 2017 & 14 & 2.00 & 1930.00 & 1 & 245 \\
\hline Avg. & 20.53 & 5.53 & 701.94 & $10^{*}$ & $301.42^{*}$ \\
\hline Std. Dev. & 7.57 & 3.60 & 658.47 & $5.42^{*}$ & $160.16^{*}$ \\
\hline
\end{tabular}

Table 1. Overall data directly available online from Springer regarding the COORDINATION conference series. To compute citations (downloads) per year, the number of citations (downloads) was divided by the number of years the publications is available since. MCP stands for "Most Cited Paper" whereas MDP stands for "Most Downloaded Paper". 


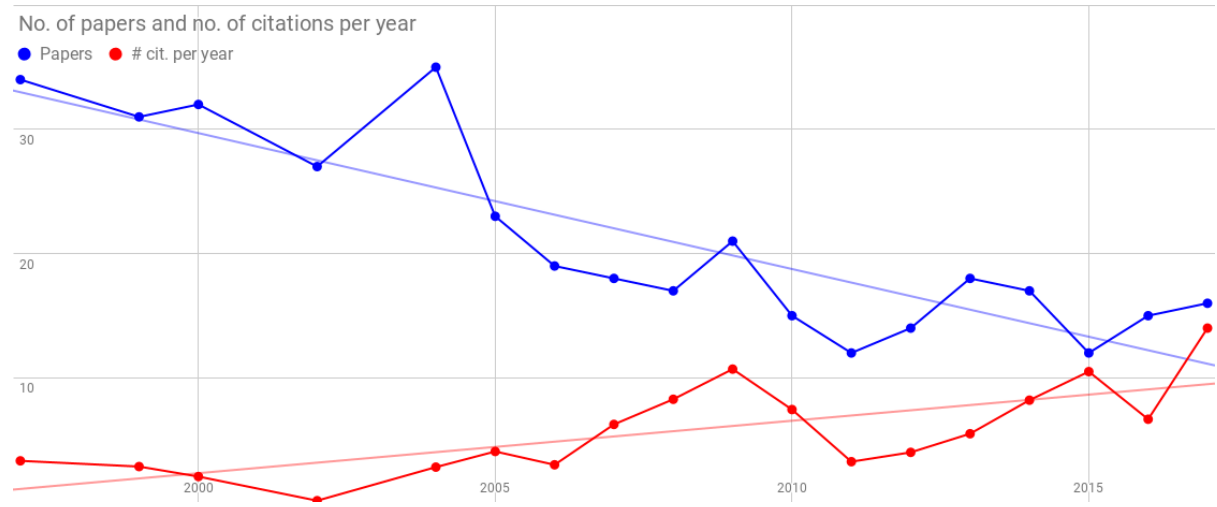

Fig. 1. Number of papers in the volume and number of citations per year (computed as described in text) of the volume.

The trend over time of the number of papers, the citations of the volumes, and their downloads, are plotted in Fig. 1 and Fig. 2 , respectively, along with their trend line. A few significant trends can be spotted in spite of the high variability between different editions of the conference. For the number of published papers, the trend is clearly descending: the first five editions featured an average of 32 papers, whereas the latest five an average of 15 . As far as the number of citations per year generated by each volume of the proceedings is concerned, a few oscillations can be observed:

- a first phase (from the $1^{\text {st }}$ edition to the $4^{\text {th }}$ ) shows a decreasing number of citations, from 3.32 down to 0.11 (the all-time-low)

1 http://link.springer.com/conference/coordination

2 http://www.bookmetrix.com/

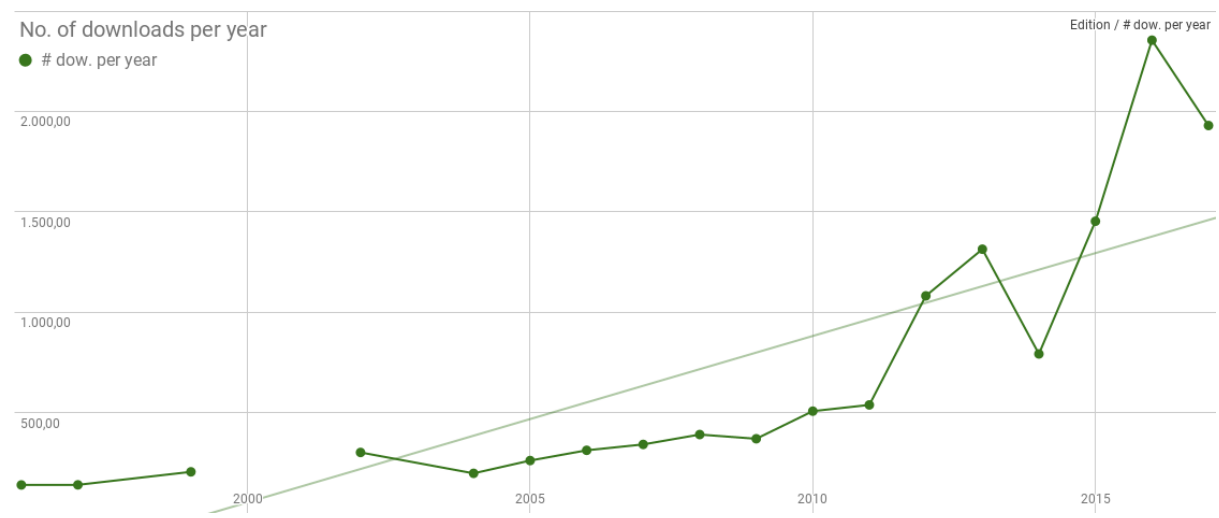

Fig. 2. Number of downloads per year (computed as described in text) of the volume. 


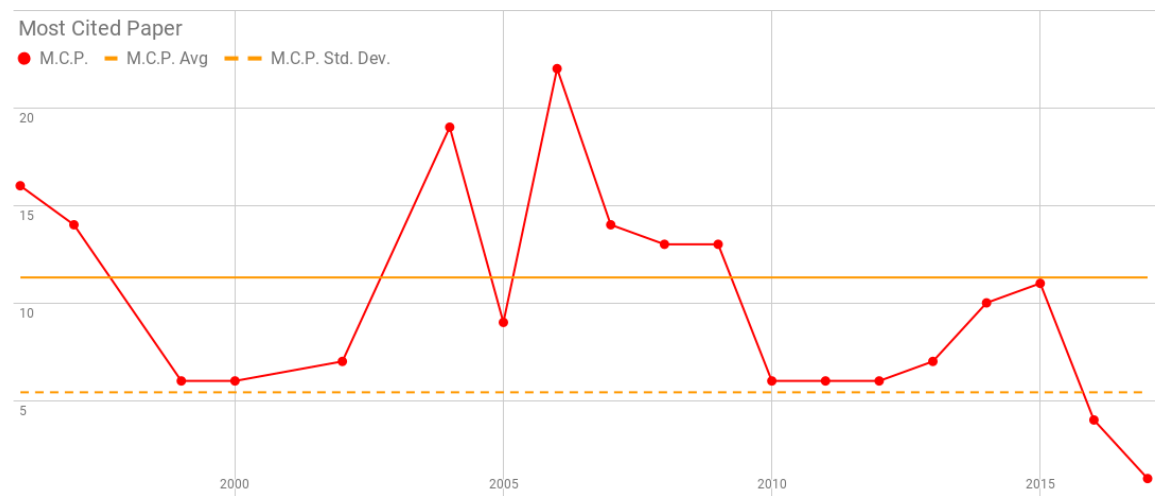

Fig. 3. Most cited paper per year with average values \& standard deviation.

- then, in a second phase (from the $5^{\text {th }}$ to the $10^{\text {th }}$ edition) the number of citations increases, up to 10.70 in 2008

- finally, after a brief fall in 2009 and 2010, the number of citations per year kept increasing up to the all-time-high of 2016 (14.00)

For the number of downloads per year, two phases can be devised out in Fig. 2 ;

- in the first period, from the $1^{\text {st }}$ edition to the $13^{\text {th }}(2011)$, the trend is quite stable, oscillating between 140 and 538.57

- in the second one instead, from 2012 up to latest edition, there is a sharp increase up to the all-time-high of 2355.00 in 2016

Finally, Fig. 3 and Fig. 4 show the most cited paper and the most downloaded paper per year, respectively. Besides noting (i) the highly irregular trend regarding the most cited papers, oscillating from 6 to 22 through approximately three epoch $\$^{3}$ (few citations during 1996-2002, more citations during 2003-2009, few

${ }^{3}$ Excluding the most recent editions, which had less time to generate citations.

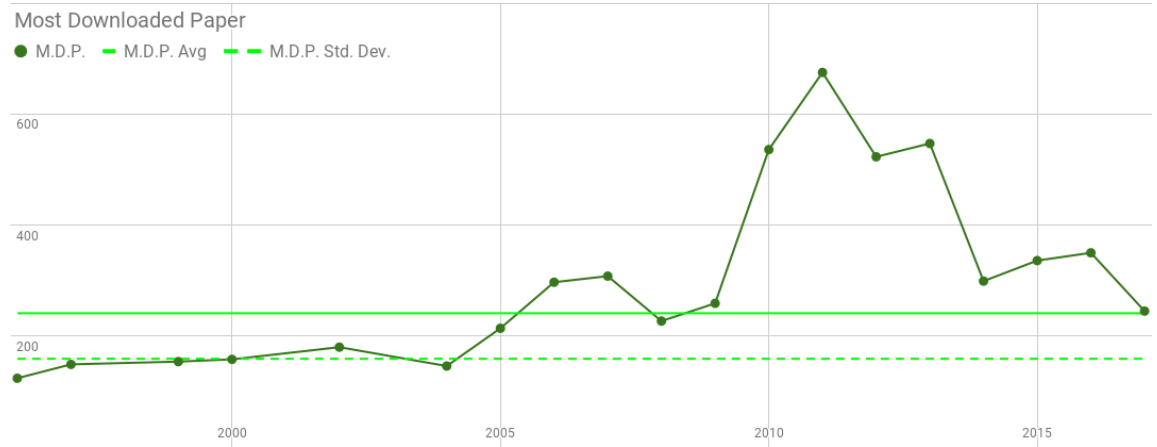

Fig. 4. Most downloaded paper per year with average values \& standard deviation. 
again during 2010-2017), and (ii) the increasing number of downloads in recent years: in the four years between 2010 and 2013 the most downloaded papers combined generated more downloads than the most downloaded papers of all the previous years combined (2281 vs. 2216), it could be interesting to check how many of such papers are related to technology, if any.

Overall, in the 19 editions of COORDINATION held until now, the most cited / downloaded paper is about technology - in the broadest acceptation of the term - in slightly less then a half of them: 7 papers amongst the most cited ones, and 8 amongst the most downloaded ones.

By extending the analysis to all the papers published in the proceedings, instead, out of all the 390 papers published, only 47 (just 12.05\%) - based on authors' inspection of the papers - convey a technological contribution. And, such an estimate is somehow optimistic, since we counted papers just for merely mentioning a technology, with no means to access it-see Table 2 .

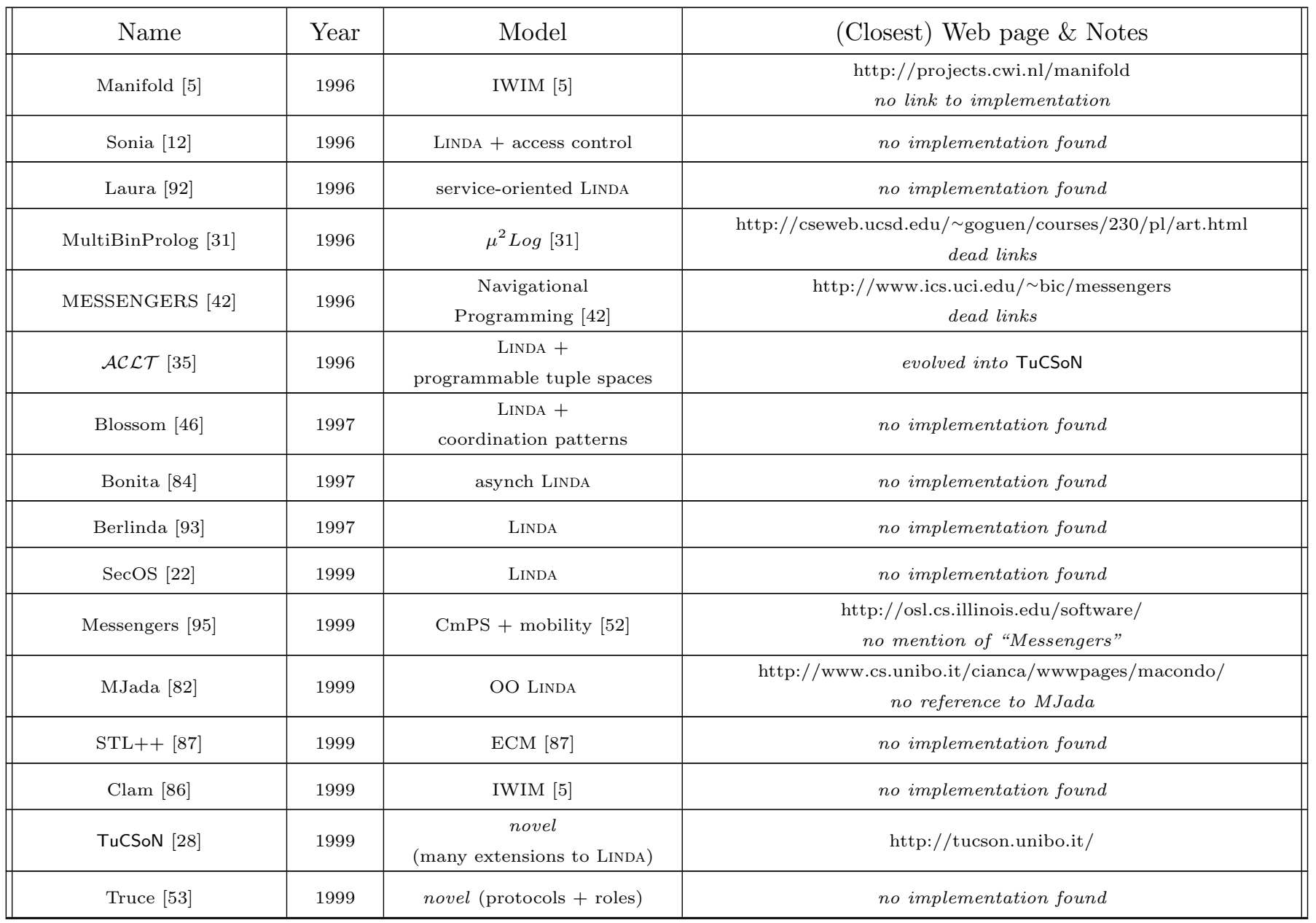




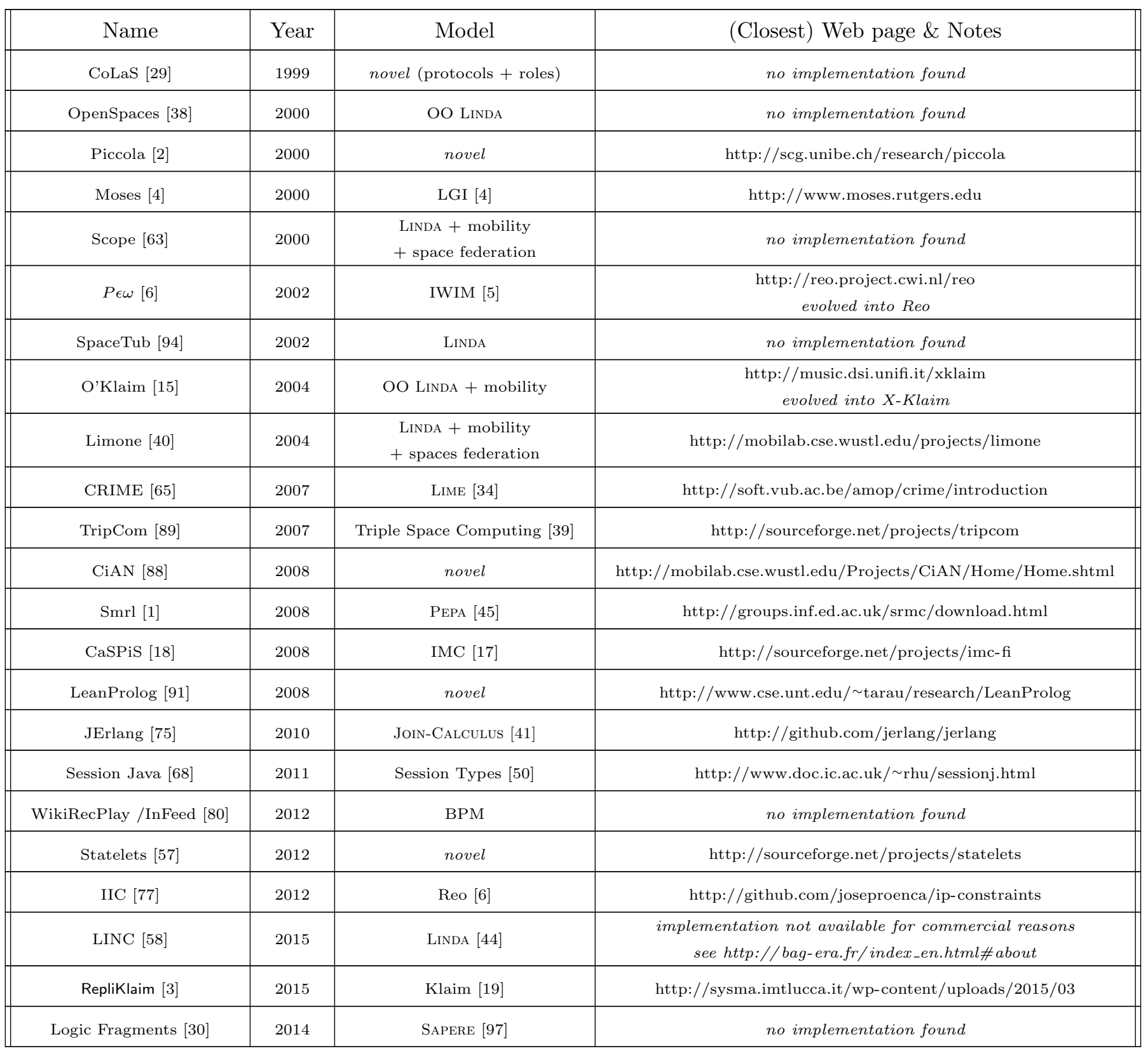

Table 2: Overview of the coordination technologies presented at COORDINATION. "Name" denotes the technology, whereas "Model" makes explicit the model taken as reference for the implementation. The last column points to the web page where the software is available - if any - and provides for additional notes. 


\subsection{Analysis of Technologies}

Table 2 provides an overview of the coordination technologies born within the COORDINATION conference series throughout the years. Only those technologies passing test $\S 2$ in Section 1.2 are included, that is, those technologies actually delivering some form of coordination services to applications - i.e. in the form of a software library with suitable API. For each technology, the original paper is referenced, the model taken as reference for implementation indicated - if any and the URL to the technology web page hosting the software given - if any is still reachable. Technologies whose corresponding software is still available - that is, those passing test $\S 3$ in Section 1.2 - are further discussed in Subsection 2.3. those with no working software found are briefly described in the following.

The early days. The first few years of COORDINATION (1996-2000) saw a flourishing of successful technologies: some of the ideas introduced back then are still alive and healthy. For instance, $\mathcal{A C L T}[35$ adopted first-order logic terms as LINDA tuples, an intuition shared by the $\mu^{2} \log$ model and its language, MultiBinProlog 31. Also, $\mathcal{A C} \mathcal{L} \mathcal{T}$ allowed agents to dynamically program tuple spaces via a specification language, enabling definition of computations to be executed in response to some events generated by interacting processes. Both features influenced the TuCSoN model and infrastructure [28], one of the few technologies to be still actively maintained nowadays.

Similarly, the IWIM coordination model and its corresponding language, MANIFOLD [5], were introduced back in 1996 and survived until present days by evolving into Reo 6. IWIM came by recognising a dichotomy between exogenous and endogenous coordination, and exploiting channel composition as a means to build increasingly complex coordination patterns.

Finally, Moses 4] was presented to the COORDINATION community as an infrastructure reifying the Low Governed Interaction (LGI) model. The technology is still alive and inspectable from its homepage, even if apparently no longer maintained. Analogously, the Piccola composition language presented in 2] clearly relies on a coordination technology which reached stability and robustness, even if it seems to be no longer maintained, too.

Besides these success stories, many other papers at that time proposed a technology, but either they only mentioned the technology without actually providing a reference to a publicly available software, or such a reference is no longer reachable (i.e. the link is dead and no reference to the software have been found on the web). For instance:

Sonia 12 - a LINDA-like approach supporting human workflows, therefore stressing aspects such as understandability of the tuple and template languages, time-awareness and timeouts, and security by means of access control

Laura 92 - a language attempting to steer LINDA towards service-orientation, where tuples can represent (formal descriptions of) service requests, offers, or results, thus enabling loosely coupled agents to cooperate by means of Linda-like primitives 
MESSENGERS 42 - following the Navigational Programming methodology [42], where strongly-mobile agents - a.k.a. Messengers - can migrate between nodes. Here, coordination is seen as "invocation [of distributed computations] and exchange of data" and it "is managed by groups of Messengers propagating autonomously through the computational network"

Blossom [46 - a LINDA variant focusing on safety, which is provided by supporting a type system for tuples and templates, and a taxonomy of access patterns to tuple spaces, aimed at supporting a sort of "least privilege" principle w.r.t. access rights of client processes

Bonita 84 - another LINDA-like technology - as its successor WCL 85 focusing on asynchronous primitives and distribution of tuple spaces, which can also migrate closer to their users

Berlinda [93 — providing a meta-model - along with a Java implementation - for instantiating different LINDA-like models

SecOS 22 - a LINDA variant focusing on security and exploring the exploitation of (a)symmetric key encryption

Messengers [95 — not to be confused with [42] despite its name, which focusses on message exchange by means of migrating actors

MJada 82 — an extension of the Jada language [25], focusing on coordinating concurrent (possibly distributed) Java agents by means of LinDA-like tuple spaces with an extended primitive set and object-oriented tuples

Clam 86] - a coordination language based on the IWIM model [5]

Truce 53 - a scripting language aimed at describing protocols to which agents must comply by enacting one or more roles

CoLaS 29] - a model and its corresponding language providing a framework where a number of participants can join interaction groups and play one or more roles within the scope of some coordination protocol. In particular, CoLaS focuses on the enforcement of coordination rules by validating and constraining participants behaviour

The millenials. After year 2000, technologies are less present amongst COORDINATION papers, but not necessarily less important. For instance, Reo made its first appearance in 2002 [6], its name written in Greek $(P \epsilon \omega)$. Reo provides an exogenous way of governing interactions between processes in a concurrent and possibly distributed system. Its strength is due to its sound semantics, enabling researchers to formally verify system evolution, as well as to the availability of software tools. The technology is indeed still alive and actively developed.

Recent implementations are more easily available on the web. Out of 22 coordination technologies, just 6 were not found on the web during the survey:

OpenSpaces 38 - focussing on the harmonisation of the LINDA model with the OOP paradigm and, in particular, with the inheritance mechanism

Scope 63] - analogously to Lime, it provides multiple distributed tuple spaces cooperating by means of local interactions when some process attempts to access a tuple, thus providing a sort of federated view on the tuple space

SpaceTub 94 - successor of Berlinda, it aims at providing a meta-framework where other LinDA-like frameworks can be reproduced 
WikiRecPlay / InFeed [80] — a pair of tools (browser extensions, no longer available) aimed at extracting and manipulating information from web applications to record them and later replay, enabling the definition of sequences of activities that can be synchronised with each other. The goal here is to augment social software with coordination capabilities.

LINC 58 - a coordination environment implementing the basic LINDA primitives - out, in, $r d$ - in a setting in which each tuple space (called bag) could implement the primitives differently (still preserving semantics), a convenient opportunity when dealing with physical devices (i.e. in the case of deployment to IoT scenarios) or legacy systems (i.e. databases). It provides transactions to alleviate to developers the burden of rolling back actions in the case of failures, and a chemical-reaction model inspired to Gamma 11 for enacting reaction rules. Several tools [59] are provided to help developers debug the rules, and to generate rules from high level specifications. The LINC software is nevertheless not publicly available because it is exploited by the Bag-Era company. Accordingly, it is not further analysed in Subsection 2.3. but it is included in Section 3 as an example of industrial success.

Logic Fragments [30] - a chemical-based and programmable coordination model likewise SAPERE [97] - to which it is inspired - enriched with a logicbased one through the notion of Logic Fragments, which are combinations of logic programs defining on-the-fly, ad-hoc chemical reactions - similar to SAPERE eco-laws - that apply on matching tuples to manipulate other tuples or to produce new Logic Fragments. The aim is to guarantee data consistency, favour knowledge representation of partial information, and support constraints satisfaction, thanks to verification of global properties enabled by the logic nature of the framework.

All the others are still publicly available, thus further analysed in next section. For instance, the O'Klaim language presented in 15$]$ evolved into the XKlaim project [16 which is still alive, even if apparently no longer maintained. Similar considerations can be made for Limone [40 and CRIME [65], which both stem from the idea of opportunistic federation of transient tuple spaces introduced by LIME [66], and improve it with additional features such as lightweightness and orientation to ambient-programming.

Analogously, the CiAN 88 model and middleware, targeting the coordination of distributed workflows over Mobile $A$ d-hoc Networks (MANETs), comes with a mature implementation, although no longer maintained. An extension to Session Java [51 is proposed in 68 to explicitly tackle synchronisation issues such as freedom from deadlock via multi-channel session primitives, Whereas the implementation was discontinued in 2011 the source code is still available from GoogleCode archive. JErlang [75, a Java-based implementation of Erlang extended with constructs borrowed from the JoIn-CALCUlus [41], appears to be no longer maintained too as explicitly stated in its home pag $5^{5}$ although a couple of implementations are still available and (partially) working.

${ }^{4}$ Year of latest commit: https://code.google.com/archive/p/sessionj

5 http://jerlang.org/ 
Also RepliKlaim [3, an implementation of KLAIM [19 aimed at optimising performance and reliability through replication of tuples and tuple spaces, received updates until 2015 as far as we know, thus appears to be discontinued. Likewise, 2015 is the year when both Statelets [57] and IIC 77] received their last known update: the former is a programming model and language aimed at integrating social context and network effects, derived from social networks analysis, as well as semantic relationships amongst shared artefacts in, i.e. groupware applications, into a single and coherent coordination model, while the latter proposes Interactive Interaction Constraints (IIC) as a novel framework to ground channel-based interaction - à la Reo - upon constraints satisfaction, interpreting the process of coordinating components as the execution of a constraints solver.

Next section briefly focuses on those technologies - that is, coordination technologies that can be actually installed and used nowadays - step $\S 4$ in Section 1.2 .

\subsection{Analysis of selected technologies}

Table 3 overviews the working technologies we were able to somewhat successfully test, that is, only those technologies listed in Table 2 which successfully surpassed test $\S 4$ described in Section 1.2 a software artefact exists and is still working.

It is worth noting that, w.r.t. Table 2, a few technologies are not included in this section despite the corresponding software is available from the reference web page therein referenced. The reason is:

- Smrl requires ancient software to run-that is, an old version of Eclipse requiring in turn an ancient version of the Java runtime (1.4)

- CaSPiS [18 (or better, JCaSPiS, namely the Java-based implementation of CaSPiS) was not found anywhere - neither in the author personal pages, nor in their account profiles on Github, nor in the web pages of the SENSORIA project mentioned in the paper. Nevertheless, the IMC model and framework allegedly grounding its implementation is still accessible ${ }^{6}$ Then we proceeded to download it looking for the CaSPiS code, without success. It is worth to be mentioned, anyway, that the IMC framework code appears to be broken, since compilation fails unless a restricted/deprecated Java API is used 7 , and even in the case of instructing the compiler to allow for it 8 the attempt to run any part of the software failed without informative error messages - just generic Java exceptions.

- LeanProlog is not usable as a coordination technology as defined in Section 1.2 it is a Prolog engine with low-level mechanisms for handling multithreading, and provides no API for general purpose coordination

- Session Java, as explicitly stated in its home page, requires an ancient version of the Java runtime to run, that is, 1.4

- Statelets is explicitly tagged as being in "pre-alpha" development stage, and, upon inspection, revealed to be only partially developed

\footnotetext{
6 https://sourceforge.net/projects/imc-fi/

7 A class uses a deprecated API, and another one requires breaking access restrictions.

${ }^{8}$ See https://goo.gl/pdWCsx.
} 


\begin{tabular}{|c|c|c|c|c|c|c|c|c|c|c|c|c|c|}
\hline 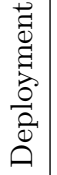 & 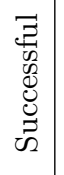 & 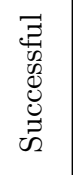 & | & 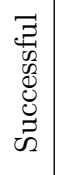 & 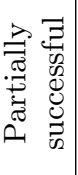 & 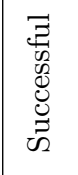 & 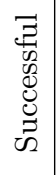 & 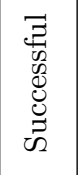 & 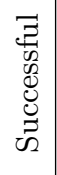 & 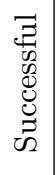 & | & | & 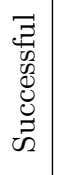 \\
\hline $\begin{array}{c}\overrightarrow{0} \\
\bar{\Xi} \\
\bar{m}\end{array}$ & 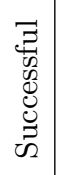 & | & 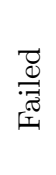 & 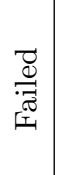 & 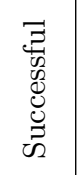 & 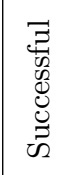 & 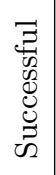 & 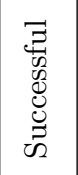 & $\mid$ & 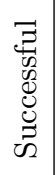 & 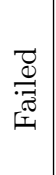 & 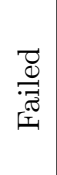 & 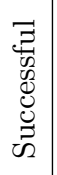 \\
\hline 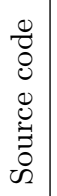 & 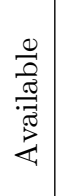 & 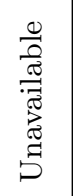 & $\begin{array}{l}\frac{0}{\pi} \\
\frac{\pi}{\pi} \\
\frac{\pi}{\pi} \\
\frac{\pi}{4}\end{array}$ & 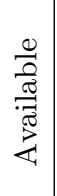 & $\begin{array}{l}\frac{0}{2} \\
\frac{\pi}{\pi} \\
\stackrel{\pi}{\pi} \\
\frac{\pi}{4}\end{array}$ & $\begin{array}{l}\frac{0}{0} \\
\frac{\pi}{\pi} \\
\frac{\pi}{\pi} \\
\frac{\pi}{4}\end{array}$ & 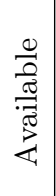 & 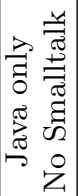 & 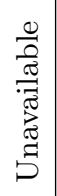 & 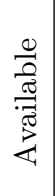 & $\begin{array}{l}\frac{0}{2} \\
\frac{\pi}{\pi} \\
\stackrel{\pi}{\pi} \\
\frac{\pi}{2}\end{array}$ & 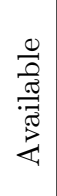 & 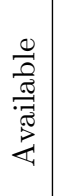 \\
\hline 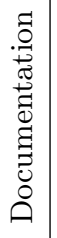 & $\begin{array}{l}\frac{0}{2} \\
\stackrel{\pi}{\pi} \\
\stackrel{\pi}{\pi} \\
\frac{\pi}{4}\end{array}$ & $\begin{array}{l}\frac{0}{0} \\
\frac{\pi}{\pi} \\
\frac{\pi}{\pi} \\
\frac{\pi}{4}\end{array}$ & $\begin{array}{l}\ddot{0} \\
0 \\
0\end{array}$ & $\begin{array}{l}\dot{0} \\
\dot{0} \\
n_{1}\end{array}$ & 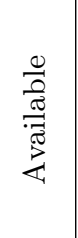 & 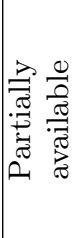 & $\begin{array}{l}\frac{0}{2} \\
\frac{\pi}{7} \\
\frac{\pi}{\pi} \\
\frac{\pi}{4}\end{array}$ & $\begin{array}{l}\frac{0}{7} \\
\frac{\pi}{\pi} \\
\frac{\pi}{\pi} \\
\frac{\pi}{4}\end{array}$ & 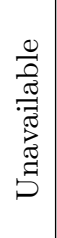 & $\begin{array}{l}\dot{\ddot{o}} \\
0\end{array}$ & $\begin{array}{l}\frac{0}{\pi} \\
\frac{\pi}{\pi} \\
\stackrel{\pi}{\pi} \\
\frac{\pi}{4}\end{array}$ & 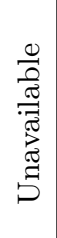 & 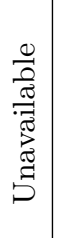 \\
\hline 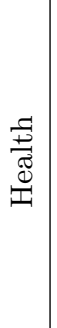 & 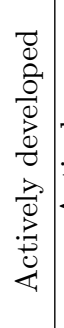 & 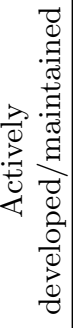 & 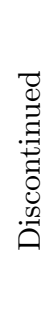 & 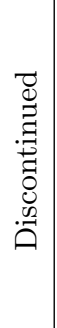 & 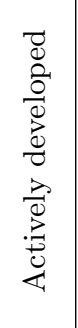 & 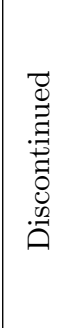 & 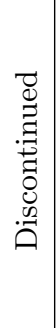 & 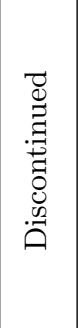 & 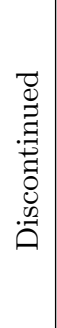 & 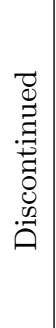 & 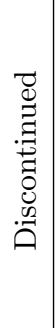 & 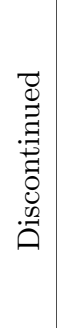 & $\stackrel{\circ}{\circ}$ \\
\hline 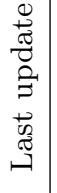 & $\stackrel{\sim}{\stackrel{\sim}{\curvearrowright}}$ & $\stackrel{\sim}{\stackrel{\sim}{\stackrel{N}{*}}}$ & $\stackrel{\sim}{\stackrel{\sim}{\circ}}$ & 足 & $\stackrel{m}{\vec{\sim}}$ & 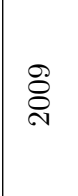 & 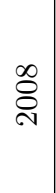 & 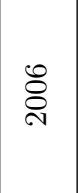 & 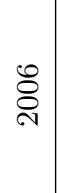 & $\begin{array}{l}\vec{\circlearrowleft} \\
\stackrel{\sim}{N}\end{array}$ & 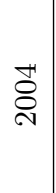 & $\underset{\sim}{\stackrel{\leftrightarrow}{8}}$ & ${ }^{\circ}$ \\
\hline 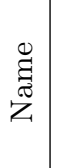 & $\begin{array}{l}z \\
0 \\
0 \\
0 \\
\Xi \\
F\end{array}$ & $\begin{array}{l}\text { D } \\
\mathbb{0}_{0} \\
\sum_{1}^{2}\end{array}$ & 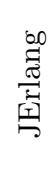 & 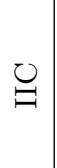 & 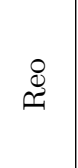 & 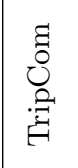 & 离 & $\begin{array}{l}\frac{\pi}{0} \\
\stackrel{0}{0} \\
\stackrel{0}{1}\end{array}$ & 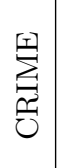 & $\frac{\pi}{\vec{a}}$ & 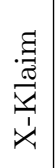 & 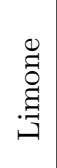 & 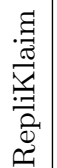 \\
\hline
\end{tabular}

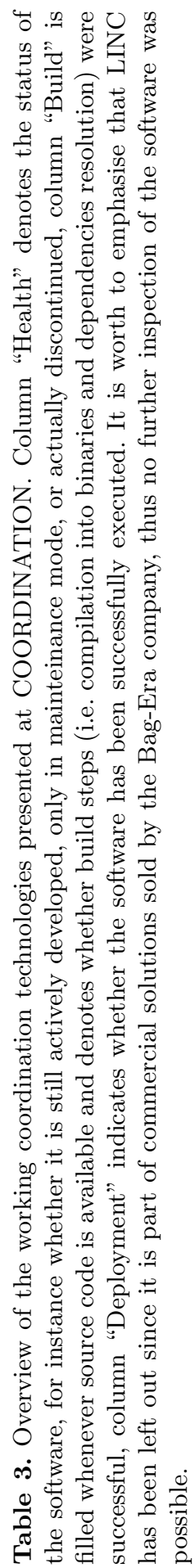


TuCSoN. Although TuCSoN 28] appeared at COORDINATION in 1999, its roots date back to the first edition of the conference, as the $\mathcal{A C} \mathcal{L} \mathcal{T}$ model [35.

TuCSoN is a coordination model adopting LINDA as its core but extending it in several ways, such as by adopting nested tuples (expressed as first-order logic terms), adding primitives (i.e. bulk [83] and uniform [60]), and replacing tuple spaces with tuple centres [71 programmable in the ReSpecT language 70]. It comes with a Java-based implementation providing coordination as a service 96 in the form of a Java library providing API and a middleware runtime, especially targeting distributed Java process but open to rational agents implemented in tuProlog [36. The TuCSoN middleware is publicly available from its home pag $£^{9}$, which provides both the binaries (a ready-to-use Java jar file) and a link to the source code repository. From there, also documentation pages are available, in the form of a usage guide and a few tutorials providing insights into specific features. Finally, a few related sub-projects are therein described too, such as TuCSoN4JADE [62 and TuCSoN4Jason [61], which are both Java libraries aimed at integrating TuCSoN with JADE 13 and Jason 21] agent runtimes, respectively, by wrapping TuCSoN services into a more convenient form which best suites those developers accustomed to programming in those platforms.

As far as technology is concerned, TuCSoN is still actively developed, being the latest commit in 2017, when also the latest related publication has been produced - an extension to the ReSpecT language and toolchain exploited to program tuple centres in TuCSoN 27]. Also, it is actively exploited as the infrastructural backbone for other projects - e.g., the smart home logic-based platform Home Manager [23 - and industrial applications - e.g., the Electronic Health Record solution 37]. Nevertheless, TuCSoN is the results of many years of active development by many different people with many different goals. Thus, despite some success stories, TuCSoN would require some substantial refactoring and refinement before it can become a truly commercially-viable product.

Moses. Moses [4] is the technology implementing the Law Governed Interaction (LGI) coordination model [64, which aims at controlling the interaction of agents interoperating on the Internet. In LGI, each agent interacts with the system by means of a controller, that is, a component exposing a fixed set of primitives allowing agents to exchange messages with other agents. The controller is in charge of intercepting invocations of primitives by interacting agents to check if they are allowed according to the law currently adopted by that controller.

Laws are shared declarative specifications dictating how the controller should react when it intercepts events of interest. Laws are expressed either in a Prologlike language or as Java classes. Each controller has its own state which can be altered by reactions to events and can influence the effect of future reactions. Non-allowed activities are technically prohibited by the controller which takes care of aborting the forbidden operation - for instance, by not forwarding a message to the intended receiver if some conditions are met.

\footnotetext{
$9 \longdiv { \text { http://tucson.unibo.it } }$
} 
The project home page ${ }^{10}$ is well-organised and provides a number of resources focussed on Moses/LGI such as reference papers, manuals, tutorials, JavaDoc, examples. The page also provides an archive with the compiled versions of the Moses middleware, the latest one dating back to 2017-suggesting that the project is actively maintained and/or developed, and representing another success story born within the COORDINATION series. We were able to successfully compile and execute the code: however, no source code is provided, and some poriontof the web page, such as the JavaDoc, are not updated w.r.t. the current Moses implementation. Finally, Moses still bounds to deprecated technologies such as Java Applets, which we believe may hinder its adoption.

JErlang. JErlang [75] is an extension of the Erlang language for concurrent and distributed programming featuring joins as the basic synchronisation constructas borrowed from the JoIn-Calculus [41]. The web page mentioned in the paper ${ }^{11}$ is no longer accessible; by searching JErlang and the authors' names on the web, a GitHub repository with the same broken reference popped up ${ }^{12}$, apparently tracking the development history of the JErlang technology. There, however, JErlang is described as an implementation of Erlang/OTP on the JVM. Also, another apparently very similar technology is therein referenced: Erjang.

Anyway, JErlang installation and usage instructions are nowhere to be found, and, when trying to build the project through the provided Maven pom.xml file, the build fails due to many errors related to obsolete dependencies - which we were not able to fix. Instead, Erjang GitHub repository - with no clue about its links to the paper - provides installation instructions, however building fails due to a Java compilation failure for a "bad class file" error ${ }^{13}$. We feel then free to declare the implementation as discontinued.

IIC. Interactive Interaction Constraints (IIC) 77] is a sort of "spin-off" of Reo introduced in 2013 [77. The original approach of implementing Reo connectors as interaction constraints is extended to allow interaction to take place also between rounds of constraints satisfaction. This extends the expressive reach of IIC beyond Reo, and makes the whole process of constraints satisfaction transactional w.r.t. observable behaviour.

The IIC software is distributed as a Scala library providing an handy syntax which eases definition of Reo-like connectors. The Scala library source code is distributed by means of a GitHub repository ${ }^{14}$ where the latest commit dates back to 2015. The library ships with a SBT configuration, allegedly supporting automatic building. Nevertheless, we were not able to reproduce the compilation process since the provided SBT configuration depends on an ancient SBT version. Therefore, we consider IIC a no longer maintained but still usable fullfledged coordination technology.

10 http://www.moses.rutgers.edu/index.html

11 https://www.doc.ic.ac.uk/ susan/jerlang/

12 Second link in "See also" section at https://github.com/jerlang/jerlang

13 Actual error is: "class file contains malformed variable arity method: [...]".

14 http://github.com/joseproenca/ip-constraints 
Reo. Reo was firstly introduced to the COORDINATION community in 6], its name in Greek letters $(P \epsilon \omega)$. Similarly to the IWIM model, Reo adopts a paradigm for exogenous coordination of concurrent and possibly distributed software components. According to the Reo model, components are the entities to be coordinated, representing the computations to be performed, while connectors are the asbtraction reifying coordination rules. The only assumption Reo makes about components is that they have a unique name and a well-defined interface in the form of a set of input ports and output ports. Conversely, connectors are composed by nodes and channels, or other connectors. A number of coordination schemes can be achieved by combining the different sorts of nodes and channels accordingly. This allows to formally specify how, when, and upon which conditions data may flow from the input to the output ports of components.

Diverse research activities originated from Reo throughout the years, mostly aimed at (i) analysing the formal properties of both Reo connectors and constraints automata [10, which are the computational model behind Reo semantics; and (ii) supporting web services orchestration 54, composition, and verification [55] by means of code generation and verification tools.

Several technologies are available from the Reo tools homepag $\notin^{15}$, collectively branded as the Extensible Coordination Tools (ECT). They consist of various Eclipse IDE plugins, such as a graphical designer for Reo connectors, and a code generator which automatically converts the graphical description into Java sources in which developers may inject applicative logic. Nevertheless, the generated code comes with no explicit support for distribution.

According to their home page, ECT are allegedly compatible with any Eclipse version starting from 3.6; while we were not able to reproduce its installation in that version (due to a dependency requiring an higher version of Eclipse), we succeeded in installing it on Eclipse version 4.7 (the latest available), but the code generator appears buggy and unstable - thus hindering further testing - because of several non-informative error messages continuously appearing when trying to use the Reo model designer - which is a required step for code generation.

The ECT source code is available from a Google Code repository ${ }^{16}$-last commit dating back to 2013. In 78 a novel implementation is proposed, named Dreams, implemented in Scala and aimed at closing the gap between Reo and distributed systems. Nevertheless, its binary distribution seems unavailable and no documentation is provided describing how to compile or use it, thus we were not able to further test this novel Dreams framework.

Trip Com. TripCom 89 is essentially a departure from the LiNDA model where the tuple space abstraction is brought towards the Semantic Web vision 47] and web-based semantic interoperability in general. The former is achieved by employing the Resource Description Framework (RDF) - that is, a representation of semantic information as a triple "subject-predicate-object" - as the tuple representation language, and by considering tuple spaces as RDF triplets

15 http://reo.project.cwi.nl/reo/wiki/Tools

16 https://code.google.com/archive/p/extensible-coordination-tools/source 
containers. Also, LiNDA primitives have been consequently re-thought under a semantics-oriented perspective - that is, by adopting an ad-hoc templating language enabling expression of semantic relationships. The latter is achieved by making triple spaces accessible on the web as SOAP-based web-services.

The implementation is hosted on a SourceForge repository ${ }^{17}$ and it is apparently discontinued, provided that the last commit dates back to 2009, and the home page lacks any sort of presentation or reference to publications or documentation. Nevertheless, the available source code appears well engineered and is well documented. It can be easily compiled into a . war file and then deployed on a Web Server (i.e. Apache Tomcat).

Once deployed, the web service is accessible via HTTP - making it is virtually interoperable with any programming language and platform - and can be tested by means of a common web browser. Additionally, the service exposes a WSDL description of the API needed to use it, which implies that a client library (aka stub) may be automatically generated using standard tools for serviceoriented architectures. Nevertheless, this WSDL description is the only form of documentation when it comes to actually interact with the web-service.

CiAN. Collaboration in Ad hoc Networks (CiAN) 88] is a Workflow Management System (WfMS) enabling users to schedule and execute their custom workflow over MANETs. It comes with a reference architecture and a middleware. The middleware keeps track of the workflow state in a distributed way, and takes into account routing of tasks' input/output data, on top of a dynamic network topology where nodes communication is likely to be opportunistic.

Workflows in CiAN are modelled as directed graphs whose vertices represent tasks, and edges represent the data-flow from a task to its successors: when a task is completed, a result value is transferred through its outgoing edges. Conditions may be specified within task definitions stating, for instance, weather a task should wait for all its inputs or just for one of them.

Users can encode their workflow descriptions via a XML-based language to be endowed to an initiator singleton node, distributing the workflow to a number of coordinator nodes in charge of allocating tasks to the available worker nodes.

While the middleware is implemented in Java, tasks logic can be implemented virtually by means of any language since CiAN only assumes the application logic to interact with the middleware by means of the SOAP protocol, which provides great interoperability. Both the middleware's source code and its compiled version are distributed through $\mathrm{CiAN}$ websit ${ }^{18}$, together with detailed documentation and some runnable examples. The source code can be easily compiled, and both the obtained binaries and those publicly available can be run smoothly. The code is well documented and engineered. Nevertheless, the source code and documentation both date back to 2008: we therefore consider the project to be mature and usable, but no longer maintained.

17 https://sourceforge.net/projects/tripcom

18 http://mobilab.cse.wustl.edu/Projects/CiAN/Software/Software.shtml 
Piccola. Piccola 2 is in its essence a composition language. It provides simple yet powerful abstractions: forms as immutable, prototype-like, key-value objects; services as functional forms which can be invoked and executed; agents as concurrent services; and channels as inter-agent communication facilities. Virtually any interaction mechanism can be built by properly composing these abstractions, such as shared variables, push and pull streams, message-passing, publish-subscribe, and so on.

Nevertheless, a limitation is due to the fact that not solely the coordination mechanisms are to be programmed with the Piccola language, but also the coordinated entities. There is thus no possibility of integration with mainstream programming languages, which is a severe limitation for adoption. Additionally, even if Piccola comes with networking capabilities virtually enabling deployment to a distributed setting, there is no middleware facility available and no opportunity with integration with others is given, which is another factor likely to hinder Piccola adoption within the scope of distributed programming and coordination.

Piccola home pag $£^{19}$ is still available and collects a number of useful resources such as documentation pages and implementation. This comes in two flavours: JPiccola, based on Java, which reached version 3.7, and SPiccola, based on Smalltalk, which reached version 0.7. Source code is provided for the Java implementation only, which correctly compiles and executes.

Nevertheless, the project appears to be discontinued, given that the last commit on the source repository dates back to 2006 .

CRIME. CRIME adheres to the Fact Spaces model, a variant of LINDA which absorbs transient federation of tuple space from Lime 66 for implementing mobile fact spaces - tuple spaces where tuples are logic facts and each tuple space is indeed a logic theory. Federated fact spaces are therefore seen as distributed knowledge bases.

In this sense, CRIME has some similarities with TuCSoN, which exploits firstorder logic tuples both as the communication items and as the coordination laws. In this context, LINDA out and in primitives collapse into logic facts assertions and retractions, respectively.

Suspensive semantics is not regarded as being essential within the scope of the Fact Spaces model, since the focus is about programming fact spaces to react to information insertion/removal (or appearance/disappearance in case of transient federation). Accordingly, users can register arbitrary logic rules by means of a Prolog-like syntax. The head of such rules represent propositions which may be proved true (activated) or unknown (deactivated) given the current knowledge base by evaluating the body of the rule. Users can then plug arbitrary application logic reacting to (de)activation of these rules.

Implementation of CRIME is available on the project home pag ${ }^{20}$ and consists of an archive shipping pre-compiled Java classes with no attached source code. The software is apparently no longer maintained: the web page has been

19 http://scg.unibe.ch/research/piccola

20 http://soft.vub.ac.be/amop/crime/introduction 
updated last in 2010, and the archive dates back to 2006. Nevertheless, the archive provides a number of example applications which have been tested and are still correctly working. No support is provided to application deployment and no documentation has been found describing how to deploy CRIME to an actual production environment.

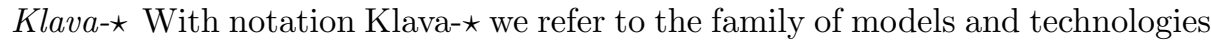
stemming from KLAIM 19 - such as O'Klaim [15 and MoMi 14 - which nowadays evolved into the X-Klaim/Klava framework 20].

$\mathrm{X}$-Klaim consists of a domain-specific language and its compiler, which produces Java code by accepting X-Klaim sources as input. The produced code exploits the Klava library in turn, that is, the Java library implementing the middleware corresponding to the KLAIM model.

The overall framework explicitly targets code mobility, thus allowing both processes and data to migrate across a network. To do so, X-Klaim and Klava provide a first-class abstraction known as locality. Localities are of two sorts: either physical, such as network nodes identifiers, or logical, such as symbolic references to network nodes having a local semantics. Each locality hosts its own tuple space, and the processes therein interacting. The LINDA primitives supported by Klava are always explicitly or implicitly related to the tuple space hosted on a specific locality. Furthermore, processes are provided with primitives enabling them to migrate from a locality to another in a strong manner, that is, along with their execution state.

Both X-Klaim and Klava are distributed by means of the KLAIM Project home pag ${ }^{21}$ providing well detailed documentation. For what concerns X-Klaim, its $\mathrm{C}++$ source code - dating back to 2004, date of the last edit, visible right below the title - is publicly available along with a self-configuring script meant to ease compilation. Nevertheless, we were not able to reproduce the compilation process on modern Linux distributions, seemingly due to some missing (and undocumented) dependency. No clues about how to fix the self-configuration process when it fails is provided, neither we were able to find some sort of documentation explicitly enumerating the compilation dependencies.

Conversely, the Klava library - actually implementing the coordination middleware - is distributed as a single . jar file containing both Java sources and the binaries. The . jar file dates back to 2004 likewise for X-Klaim, so it is apparently no longer developed, but further testing showed how the Klava library is still functioning, since it is self-contained and targets Java versions $1.4+$.

Limone. Limone [40] is a model and middleware meant to improve scalability and security in Lime [66] through access control, and explicitly targeting distributed mobile systems and, in particular, agents roaming across ad-hoc networks built on top of opportunistically interconnected mobile devices.

Once two or more devices enter within their respective communication range and thus establish a connection, the agents running on top of them are (potentially) enabled to interact by means of transient sharing of their own tuple

21 http://music.dsi.unifi.it/klaim.html 
spaces. But, for some agents to be actually able to communicate, Limone states they should specify their engagement policies. An agent $A$ 's engagement policy determines which agents are allowed to interact with it and to which extent, that is, which primitives are allowed to be invoked. Agents satisfying the policy are registered within $A$ 's acquaintance list. So, each agent only has to care about its acquaintance list, thus reducing the bandwidth requirements for the middleware.

A reactive programming mechanism completes the picture, enabling agents to inform their peer about their interest in tuples matching a given template, in order to be informed when such tuples becomes available.

The Limone technology is distributed by means of the project web page ${ }^{22}$ in the form of a compressed archive containing the Java source code (dated back in 2004) and a Makefile for automatic build. Nevertheless, the code strictly requires to be compiled against a Java version prior to 1.5, and modern Java compilers do not support such an ancient version 23 . For these reasons, we could not proceed to further test the technology and we consider it to be no longer maintained nor actually usable.

RepliKlaim. RepliKlaim 3 is a variant of Klaim [19 introducing first-class abstractions and mechanisms to deal with data locality and consistency, so as to give programmers the ability to explicitly account for and tackle these aspects when developing parallel computing applications. Specifically, the idea is to let the programmer specify and coordinate replication of data, and operate on replicas with a configurable level of consistency. This enables the programmer to adapt data distribution and locality to the needs of the application at hand, especially with the goal of improving performance in terms of concurrency level and data access speed - in spite of latencies due to distribution.

Most of the abstractions and mechanisms, as well as syntax elements and semantics, of RepliKlaim are exactly as in Klaim, such as data repositories, processes, locations, and many actions. When due, actions are extended to explicitly deal with replication aspects, such as in the case of an out primitive putting multiple copies of the same tuple in multiple localities, or an in primitive removing all replicas from all locations at once. Also, various degrees of consistency among replicas in the same or different locations are achieved depending on whether primitives are synchronous (namely, atomically executed) or asynchronous.

There exists a prototype implementation of RepliKlaim on top of Klava, the Java implementation of Klaim, available for direct download from a URL ${ }^{24}$ given in its companion paper [3]. From there, a .rar archive is provided, containing a version of Klava and the source files implementing RepliKlaim, which can be easily compiled and run successfully.

Nevertheless, as stated in the paper describing RepliKlaim, its implementation currently relies on encoding its model in the standard Klaim model, thus,

22 http://mobilab.cse.wustl.edu/projects/limone

23 As stated here: https://docs.oracle.com/javase/9/tools/javac.htm\#JSWOR627

24 http://sysma.imtlucca.it/wp-content/uploads/2015/03/RepliKlaim-test-examples.

rar 


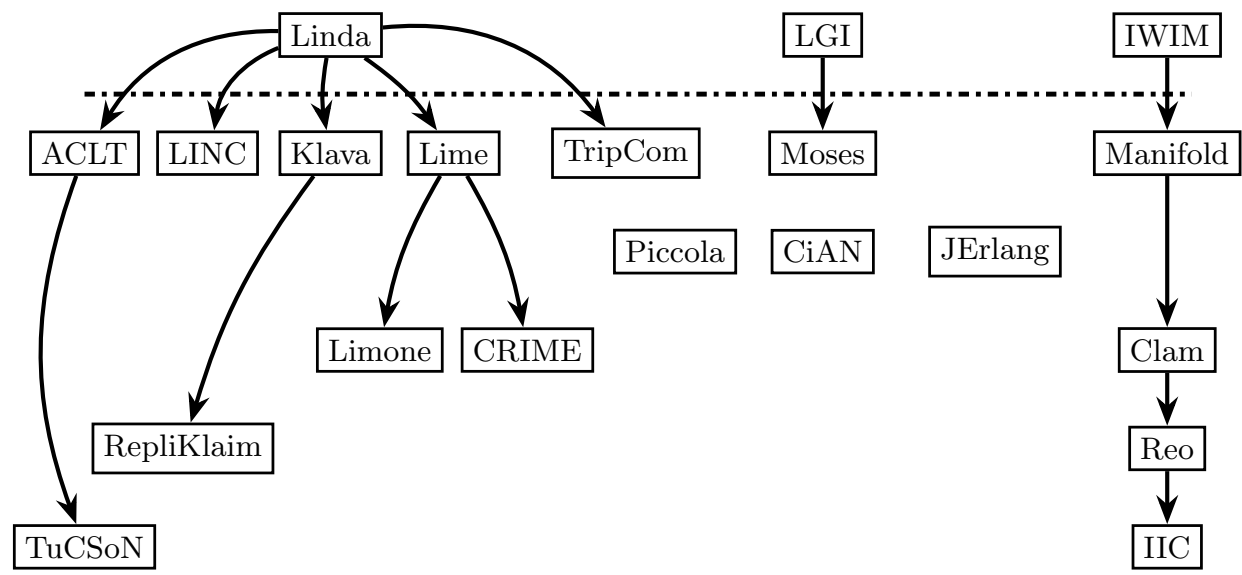

Fig. 5. Lines of evolution of selected technologies (below the dashed line), as stemming from a few archetypal coordination model (above the dashed line).

on the practical side the code provided only features examples about how to translate RepliKlaim primitives into Klava. No higher-level API directly providing to developers the replica-oriented operations of RepliKlaim is provided. In other words, there exists no RepliKlaim Java library which can be imported to other java projects in order to exploit its provided coordination services.

\section{Discussion}

In this section we aim at providing further insights about the technologies described in Subsection 2.3, especially to understand (i) whether they stem from a common archetypal coordination framework (Fig. 5), (ii) their relationships in terms of the features they provide (Fig. 6), and (iii) which goal mostly motivated their development and which application scenario they mostly target (Fig. 7).

A family tree. Fig. 5 depicts a sort of "family tree" of the selected coordination technologies, emphasising how they stem from a few archetypal coordination models/languages, and how they are built on each other. It makes thus apparent how most of the technologies still available stem from two archetypal models: LINDA [44 and IWIM 5]. Nevertheless, whereas in the case of LindA many heterogeneous extensions have been proposed throughout the years, focussing on different features and thus diverging from LINDA in many diverse ways, the evolution of the IWIM model appears much more homogeneous, featuring descendants which "linearly" extend their ancestors' features. Summing up, from LINDA stem the TuCSoN family, the Klaim [19] family - including Klava and RepliKlaim -, the LIME 74 family - with Limone and CRIME -, besides the lone runners LINC and TripCom, whereas from the IWIM root stems the Reo family - completed by Manifold, Clam, and the latest extension IIC. 
Apart from these two big family trees, we have the LGI model, along with its implementation, Moses, and a small group of "lone runners" with unique features: Piccola, CiAN, and JErlang. While the former inspired some features of technologies stemming from other models - for instance, its programmable laws inspired essentially any other technology or model having reactive rules of some sort, such as LINC -, the latter remained mostly confined to itself.

It is interesting to notice how "the IWIM family" and "the LINDA family" remained well-isolated one from each other over all these years. Whereas this can be easily attributed to the fundamental difference in the approach to coordination they have - data-driven vs. control-driven, as also emphasised in Fig. 6 on page 23 - it seems odd that nobody tried to somewhat integrate these two extremely successful coordination models, in an attempt to improve the state of art by cherry-picking a few features from both to create a novel, hybrid coordination model [69], with "the best of two worlds". To some extent, the TuCSoN model, along with its coordination language, ReSpecT, pursues this path: ReSpecT in fact can be regarded as a data-driven model because coordination is based on availability of tuples, as in LiNDA, but, at the same time, coordination policies are enforced by declarative specifications which control the way in which the coordination medium behaves, thus, ultimately, how the coordinated components interact - as typical for control-driven models like IWIM.

We believe that the path toward integration could be the key in further perfecting and improving coordination models and languages, by complementing data-driven models elegance and flexibility with control-driven models finegrained control and predictability.

Families marriage. Fig. 6 enriches the family tree just described with relationships indicating differences (red and green arrows) and similarities (blue arrows) in features provided - notice that w.r.t. Fig. 5 Piccola, CiAN, and JErlang have been removed because they are so unique that no clear relationship may be found with other technologies. As already mentioned for Fig. 5, Linda has been taken as the common ground for many technologies which are instead very heterogeneous in the aim pursued: if $\mathcal{A C} \mathcal{L} \mathcal{T}$, TuCSoN, and LINC have a LINDA core enriched with many other features - such as programmability, transactionality, and novel primitives -, the Klaim family and the LIME one diverge more, by changing the way in which primitives behave - as in the case of localities in Klaim -, or the way in which the interacting processes see each others' tuple spaces - as for Lime transient federation.

Nevertheless, technologies which may appear as being far apart from each other have interesting similarities, as in the case of the interaction rules of LGI, thus Moses, which strongly resembles $\mathcal{A C L}$ T and TuCSoN reactions, or the fact that both the Reo family and Moses are based on message passing. Or, the fact that both CRIME and TuCSoN rely on logic tuples so as to leverage on the inference capabilities of interacting agents, while Reo and both Lime and Klaim take into account mobility of processes and coordination abstractions (tuple spaces vs. channels) as a first-class citizen. 


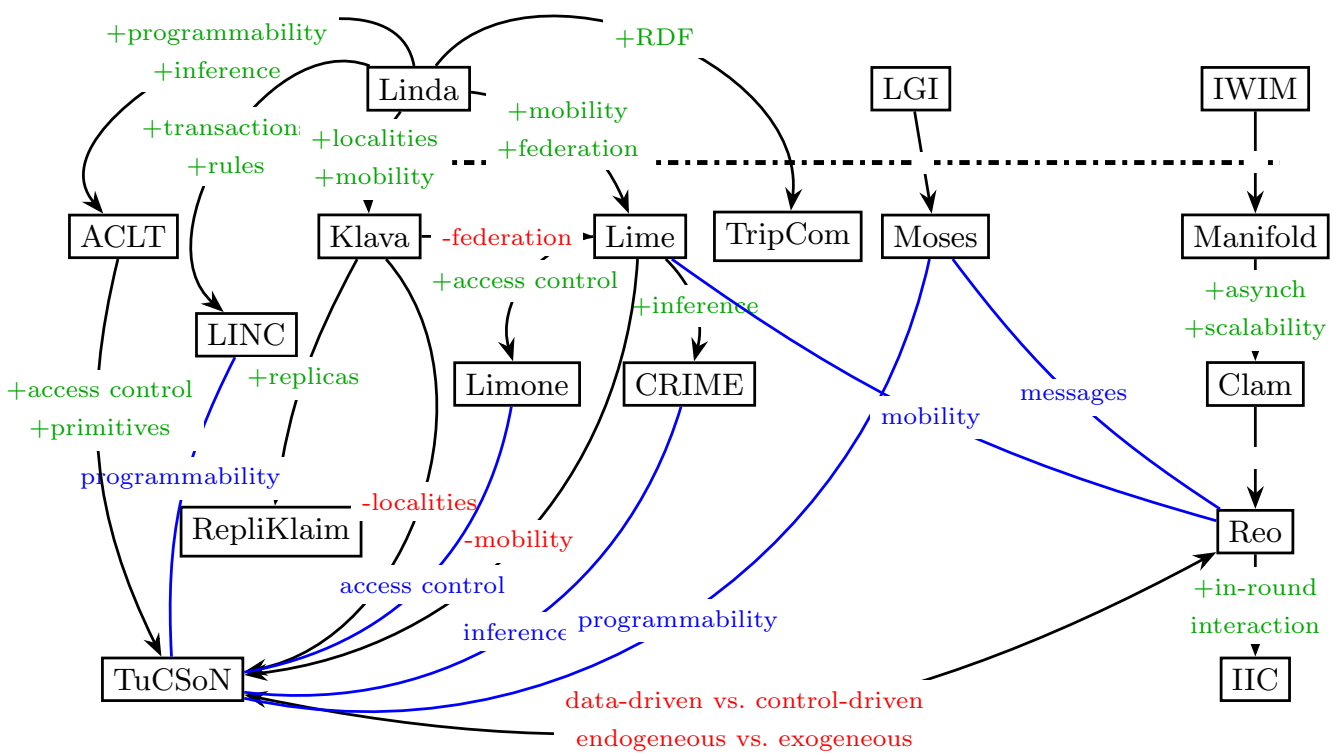

Fig. 6. Main differences (in green and red) and similarities (in blue) amongst selected technologies. Arrows indicate what it takes (in green, add something; in red, remove something) to go from one technology (the source) to another (the destination).

It is worth emphasising here that Fig. 6 highlights the features to which more attention has been devoted throughout the years: programmability, access control, and mobility. We believe that these features, possibly extended with scalability and inference capabilities, are crucial for widening applicability of coordination technologies to real-world scenarios. For instance, the Internet of Things (IoT) 9] - along with its variants Web of Things [48 and Internet of Intelligent Things 8 - is a very good fit for testing coordination technologies, and requires precisely the aforementioned features.

Goals $\&$ preferred scenarios. Finally, Fig. 7 relates the selected technologies with the main aim pursued which motivates their extension in a particular direction, along with the applications scenario they best target.

From the description of the selected technologies we gathered, two are the main goals motivating their evolution: (i) providing flexibility so as to deal with the majority of heterogeneous application scenarios possible, and (ii) focussing on first-class abstractions for better supporting space-awareness of both the coordination abstractions and the interacting processes.

In fact, TuCSoN / $\mathcal{A C} \mathcal{L} \mathcal{T}$, LINC, and Moses all provide means to somewhat program the coordinative behaviour of the coordination medium, thus aim at making it configurable, adaptable, malleable, even at run-time, and/or provide 


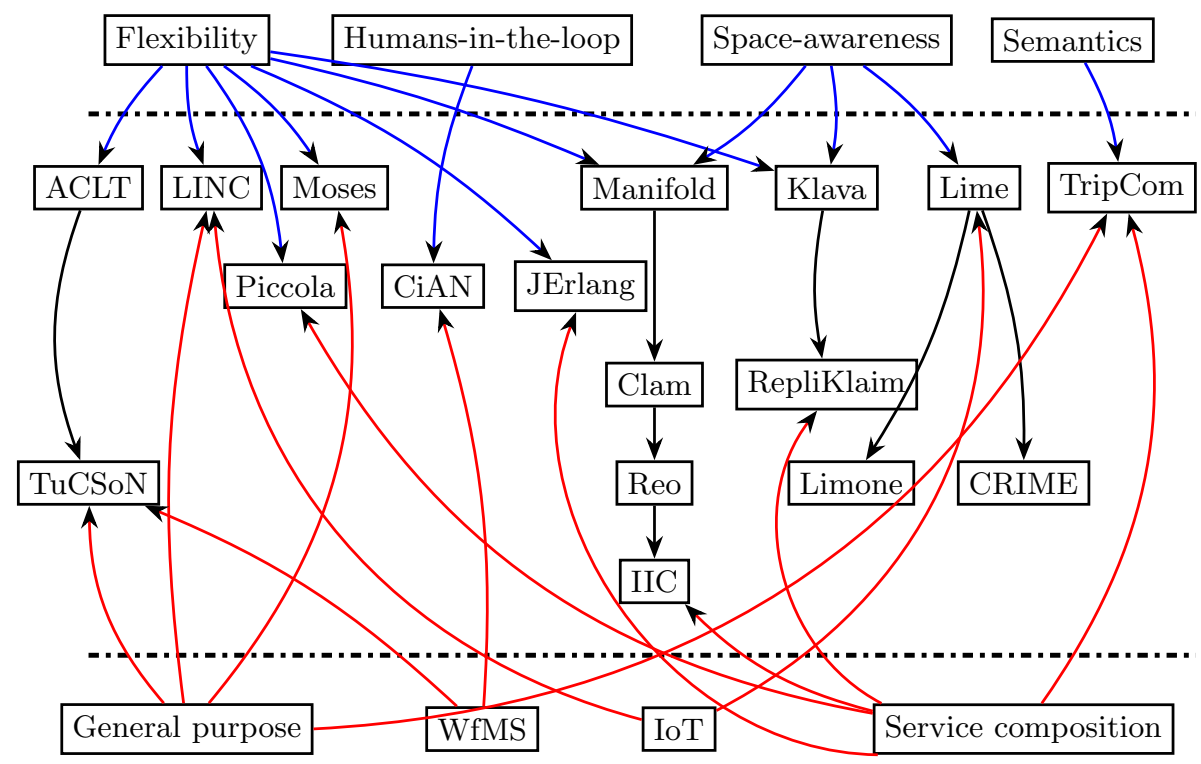

Fig. 7. Selected technologies per main goal pursued (top, blue arrows) and preferred application scenario (bottom, red arrows).

additional coordination primitives to expand the expressive reach of the coordination technology. The Klaim family, the Reo family, and the Lime family instead, are geared toward some forms of space-awareness, be it by promoting mobility or by providing location-sensitive primitives.

Besides these, two more main goals can be devised, peculiar to specific technologies: (iii) supporting humans-in-the-loop, in the case of CiAN, and (iv) provide a semantic representation of data items, in the case of TripCom.

About the application scenarios explicitly declared as of particular interest for the technology, the most prominent one is service composition, which is especially interesting for Piccola, JErlang, the Reo family, the Klaim family, and TripCom - besides being naturally applicable to all other technologies too. Then, whereas technologies such as LINC and the Lime family are mainly tailored to the IoT landscape, being meant to cope with the requirements posed by small, possibly portable, possibly embedded devices with low resources, Workflow Management (WfMS) is peculiar to CiAN, while also considered by TuCSoN 79]. Besides these application scenarios, there are many technologies without a specific focus, although they have been applied to many different ones, such as TuCSoN itself, LINC, Moses, and TripCom: these have been associated with the generic "General purpose" scenario.

We believe that the goals and application scenarios just highlighted strengthen our previous consideration that the IoT could be the "killer-app" for coordination technologies. In fact, flexibility (there including programmability and configurability), space-awareness (there including mobility and location-awareness), and 
semantics (there including interoperability of data representation formats) are all necessary ingredients for any non-trivial IoT deployment: the former helps in dealing with uncertainty and unpredictability typical of the IoT scenarios, the latter is required for building open IoT systems, and some form of spaceawareness is a common feature of many IoT deployments, from retail to industry 4.0. Also, the fact that service composition has been already thoroughly explored is a great advantage and the perfect starting point for tackling IoT challenges: both the IoT and the Web of Things vision foster a world where connected objects provide and consume services, which can be composed in increasingly high-level ones.

\section{Conclusion}

The main aim of this paper is to provide insights about the state-of-the-art of coordination technologies after twenty years of the COORDINATION conference series, and to stimulate informed discussion about future perspectives. Overall, apart from some notable success stories - i.e. the commercial success of LINC along with the active development of TuCSoN and Reo - most coordination technologies have gone through a rapid and effective development at the time they were presented, then lacked further improvements or even maintenance of its usability, thus never reached a wider audience - i.e. outside the COORDINATION community or in the industry. Obviously, something also happens outside the COORDINATION boundaries. For instance, coordination technologies are surveyed in 73 , whereas 81 focuses on tuple-based technologies. However, mostly of the technological developments reported here just happened after those survey were published, in 200172 .

Although we acknowledge that researchers are usually mostly concerned with providing scientifically-relevant models rather than production-ready software, we also believe that backing up models and languages with more then proof-ofconcept software is crucial to promote wider adoption of both the technology itself and the models, which in turn may provide invaluable feedback to researchers for further developing and tuning models. The next decade will probably tell us more about the actual role of coordination technologies in the development of forthcoming application scenarios: the IoT, for instance, is at the "peak of inflated expectations" according to Gartner's hype cycle for 2017, and is expected to reach the plateau in 2 to 5 years. This means the time is ripe for pushing forward the development of coordination technologies, so as to have them ready when the IoT will be mature enough to actually benefit from their added value.

Besides coordination technologies, we believe the COORDINATION conference is quite healthy: although the number of published papers is decreasing, citations and downloads grows (modulo too recent years), and contributions conveying technological advancements still represent almost a half of all the contributions. 


\section{References}

1. Abreu, J., Fiadeiro, J.L.: A coordination model for service-oriented interactions. In: Lea and Zavattaro [56], pp. 1-16

2. Achermann, F., Kneubuehl, S., Nierstrasz, O.: Scripting coordination styles. In: Porto and Roman [6], pp. 19-35

3. Andrić, M., De Nicola, R., Lafuente, A.L.: Replica-based high-performance tuple space computing. In: Holvoet and Viroli [49, pp. 3-18

4. Ao, X., Minsky, N., Nguyen, T.D., Ungureanu, V.: Law-Governed Internet communities. In: Porto and Roman 76], pp. 133-147

5. Arbab, F.: The IWIM model for coordination of concurrent activities. In: Ciancarini and Hankin 24], pp. 34-56

6. Arbab, F., Mavaddat, F.: Coordination through channel composition. In: Arbab and Talcott 7], pp. 22-39

7. Arbab, F., Talcott, C. (eds.): Coordination Models and Languages. 5th International Conference, COORDINATION 2002 York, UK, April 8-11, 2002 Proceedings, LNCS, vol. 2315. Springer (2002)

8. Arsénio, A., Serra, H., Francisco, R., Nabais, F., Andrade, J., Serrano, E.: Internet of Intelligent Things: Bringing artificial intelligence into things and communication networks. In: Xhafa, F., Bessis, N. (eds.) Inter-cooperative Collective Intelligence: Techniques and Applications, SCI, vol. 495, pp. 1-37. Springer (2014)

9. Atzori, L., Iera, A., Morabito, G.: The Internet of Things: A survey. Computer Networks 54(15), 2787-2805 (2010)

10. Baier, C., Sirjani, M., Arbab, F., Rutten, J.: Modeling component connectors in reo by constraint automata. Science of Computer Programming 61(2), 75-113 (Jul 2006)

11. Banătre, J.P., Fradet, P., Le Métayer, D.: Gamma and the chemical reaction model: Fifteen years after. In: Calude, C.S., Păun, G., Rozenberg, G., Salomaa, A. (eds.) Multiset Processing, LNCS, vol. 2235, pp. 17-44. Springer (2001)

12. Banville, M.: Sonia: An adaptation of Linda for coordination of activities in organizations. In: Ciancarini and Hankin 24], pp. 57-74

13. Bellifemine, F.L., Poggi, A., Rimassa, G.: JADE-a FIPA-compliant agent framework. In: 4th International Conference and Exhibition on the Practical Application of Intelligent Agents and Multi-Agent Technology (PAAM-99). pp. 97-108 (1999)

14. Bettini, L., Bono, V., Venneri, B.: Coordinating mobile object-oriented code. In: Arbab and Talcott 7], pp. 56-71

15. Bettini, L., Bono, V., Venneri, B.: O'Klaim: A coordination language with mobile mixins. In: De Nicola et al. 33. pp. 20-37

16. Bettini, L., De Nicola, R.: Mobile distributed programming in X-KLAIM. In: Bernardo, M., Bogliolo, A. (eds.) Formal Methods for Mobile Computing, LNCS, vol. 3465, pp. 29-68. Springer (2005)

17. Bettini, L., De Nicola, R., Falassi, D., Lacoste, M., Lopes, L., Oliveira, L., Paulino, H., Vasconcelos, V.T.: A software framework for rapid prototyping of run-time systems for mobile calculi. In: Priami, C., Quaglia, P. (eds.) Global Computing, pp. 179-207. Springer (2005)

18. Bettini, L., De Nicola, R., Loreti, M.: Implementing session centered calculi. In: Lea and Zavattaro [56, pp. 17-32

19. Bettini, L., Loreti, M., Pugliese, R.: An infrastructure language for open nets. In: 2002 ACM Symposium on Applied Computing (SAC 2002). pp. 373-377. ACM, New York, NY, USA (2002) 
20. Bettini, L., Nicola, R.D., Pugliese, R.: X-Klaim and Klava: Programming mobile code. Electronic Notes in Theoretical Computer Science 62, 24-37 (2002)

21. Bordini, R.H., Hübner, J.F., Wooldridge, M.J.: Programming Multi-Agent Systems in AgentSpeak using Jason. John Wiley \& Sons, Ltd (Oct 2007)

22. Bryce, C., Oriola, M., Vitck, J.: A coordination model for agents based on secure spaces. In: Ciancarini and Wolf 26], pp. 4-20

23. Calegari, R., Denti, E.: Building Smart Spaces on the Home Manager platform. ALP Newsletter (Dec 2016)

24. Ciancarini, P., Hankin, C. (eds.): Coordination Languages and Models. 1st International Conference, COORDINATION '96 Cesena, Italy, April 15-17, 1996 Proceedings, LNCS, vol. 1061. Springer (1996)

25. Ciancarini, P., Rossi, D.: Jada: Coordination and communication for Java agents. In: Vitek, J., Tschudin, C. (eds.) Mobile Object Systems Towards the Programmable Internet, LNCS, vol. 1222, pp. 213-226. Springer (1997)

26. Ciancarini, P., Wolf, A.L. (eds.): Coordination Languages and Models. 3rd International Conference COORDINATION'99 Amsterdam, The Netherlands, April 26-28, 1999 Proceedings, LNCS, vol. 1594. Springer (1999)

27. Ciatto, G., Mariani, S., Omicini, A.: Programming the interaction space effectively with ReSpecTX. In: Ivanović, M., et al. (eds.) Intelligent Distributed Computing XI, pp. 89-101. Springer International Publishing (2018)

28. Cremonini, M., Omicini, A., Zambonelli, F.: Coordination in context: Authentication, authorisation and topology in mobile agent applications. In: Ciancarini and Wolf [26], pp. 416-416

29. Cruz, J.C., Ducasse, S.: A group based approach for coordinating active objects. In: Ciancarini and Wolf 26], pp. 355-370

30. De Angelis, F.L., Di Marzo Serugendo, G.: Logic Fragments: A coordination model based on logic inference. In: Holvoet and Viroli [49], pp. 35-48

31. De Bosschere, K., Jacquet, J.M.: $\mu 2 \mathrm{Log}$ : Towards remote coordination. In: Ciancarini and Hankin 24, pp. 142-159

32. De Meuter, W., Roman, G.C. (eds.): Coordination Models and Languages. 13th International Conference, COORDINATION 2011, Reykjavik, Iceland, June 6-9, 2011. Proceedings, LNCS, vol. 6721. Springer (2011)

33. De Nicola, R., Ferrari, G.L., Meredith, G. (eds.): Coordination Models and Languages. 6th International Conference, COORDINATION 2004 Pisa Italy, February 24-27, 2004 Proceedings, LNCS, vol. 2949. Springer (2004)

34. Dedecker, J., Van Cutsem, T., Mostinckx, S., D'Hondt, T., De Meuter, W.: Ambient-oriented programming. In: Companion to the 20th Annual ACM SIGPLAN Conference on Object-oriented Programming, Systems, Languages, and Applications. pp. 31-40. OOPSLA '05, ACM, New York, NY, USA (2005)

35. Denti, E., Natali, A., Omicini, A., Venuti, M.: An extensible framework for the development of coordinated applications. In: Ciancarini and Hankin 24], pp. 305320

36. Denti, E., Omicini, A., Ricci, A.: tuProlog: A light-weight Prolog for Internet applications and infrastructures. In: Ramakrishnan, I. (ed.) Practical Aspects of Declarative Languages, LNCS, vol. 1990, pp. 184-198. Springer (2001)

37. Dubovitskaya, A., Urovi, V., Barba, I., Aberer, K., Schumacher, M.I.: A multiagent system for dynamic data aggregation in medical research. BioMed Research International 2016 (2016)

38. Ducasse, S., Hofmann, T., Nierstrasz, O.: Openspaces: An object-oriented framework for reconfigurable coordination spaces. In: Porto and Roman [76, pp. 1-18 
39. Fensel, D.: Triple-space computing: Semantic web services based on persistent publication of information. In: Aagesen, F.A., Anutariya, C., Wuwongse, V. (eds.) Intelligence in Communication Systems, LNCS, vol. 3283, pp. 43-53. Springer (2004)

40. Fok, C.L., Roman, G.C., Hackmann, G.: A lightweight coordination middleware for mobile computing. In: De Nicola et al. 33], pp. 135-151

41. Fournet, C., Gonthier, G.: The reflexive CHAM and the Join-calculus. In: 23rd ACM SIGPLAN-SIGACT Symposium on Principles of Programming Languages. pp. 372-385. ACM (1996)

42. Fukuda, M., Bic, L.F., Dillencourt, M.B., Merchant, F.: Intra- and inter-object coordination with MESSENGERS. In: Ciancarini and Hankin [24], pp. 179-196

43. Garlan, D., Le Métayer, D. (eds.): Coordination Languages and Models. 2nd International Conference COORDINATION '97 Berlin, Germany, September 1-3, 1997 Proceedings, LNCS, vol. 1282. Springer (1997)

44. Gelernter, D.: Generative communication in Linda. ACM Transactions on Programming Languages and Systems (TOPLAS) 7(1), 80-112 (Jan 1985)

45. Gilmore, S., Hillston, J.: The PEPA workbench: A tool to support a process algebra-based approach to performance modelling. In: Haring, G., Kotsis, G. (eds.) Computer Performance Evaluation Modelling Techniques and Tools, LNCS, vol. 794, pp. 353-368. Springer (1994)

46. van der Goot, R., Schaeffer, J., Wilson, G.V.: Safer tuple spaces. In: Garlan and Le Métayer 43], pp. 289-301

47. Hendler, J.A.: Agents and the Semantic Web. IEEE Intelligent Systems 16(2), 30-37 (Mar 2001)

48. Heuer, J., Hund, J., Pfaff, O.: Toward the Web of Things: Applying Web technologies to the physical world. Computer 48(5), 34-42 (May 2015)

49. Holvoet, T., Viroli, M. (eds.): Coordination Models and Languages. 17th International Conference, COORDINATION 2015, Grenoble, France, June 2-4, 2015, Proceedings, LNCS, vol. 9037. Springer (2015)

50. Honda, K., Vasconcelos, V.T., Kubo, M.: Language primitives and type discipline for structured communication-based programming. In: Hankin, C. (ed.) Programming Languages and Systems, LNCS, vol. 1381, pp. 122-138. Springer (1998)

51. Hu, R., Yoshida, N., Honda, K.: Session-based distributed programming in Java. In: Vitek, J. (ed.) ECOOP 2008 - Object-Oriented Programming, LNCS, vol. 5142, pp. 516-541. Springer (2008)

52. Jagannathan, S.: Communication-passing style for coordination languages. In: Garlan and Le Métayer [43, pp. 131-149

53. Jamison, W.C., Lea, D.: TRUCE: Agent coordination through concurrent interpretation of role-based protocols. In: Ciancarini and Wolf [26], pp. 384-398

54. Jongmans, S.S.T.Q., Santini, F., Sargolzaei, M., Arbab, F., Afsarmanesh, H.: Orchestrating web services using Reo: from circuits and behaviors to automatically generated code. Service Oriented Computing and Applications 8(4), 277-297 (Dec 2014)

55. Kokash, N., Krause, C., de Vink, E.: Reo + mCRL2: A framework for modelchecking dataflow in service compositions. Formal Aspects of Computing 24(2), 187-216 (Mar 2012)

56. Lea, D., Zavattaro, G. (eds.): Coordination Models and Languages. 10th International Conference, COORDINATION 2008, Oslo, Norway, June 4-6, 2008. Proceedings, LNCS, vol. 5052. Springer (2008)

57. Liptchinsky, V., Khazankin, R., Truong, H.L., Dustdar, S.: Statelets: Coordination of social collaboration processes. In: Sirjani [90], pp. 1-16 
58. Louvel, M., Pacull, F.: LINC: A compact yet powerful coordination environment. In: Kühn, E., Pugliese, R. (eds.) Coordination Models and Languages, LNCS, vol. 8459, pp. 83-98. Springer (2014)

59. Louvel, M., Pacull, F., Rutten, E., Sylla, A.N.: Development tools for rule-based coordination programming in LINC. In: Jacquet, J.M., Massink, M. (eds.) Coordination Models and Languages, LNCS, vol. 10319, pp. 78-96. Springer (2017)

60. Mariani, S., Omicini, A.: Coordination mechanisms for the modelling and simulation of stochastic systems: The case of uniform primitives. SCS M\&S Magazine $\operatorname{IV}(3), 6-25$ (Dec 2014)

61. Mariani, S., Omicini, A.: Multi-paradigm coordination for MAS: Integrating heterogeneous coordination approaches in MAS technologies. In: Santoro, C., Messina, F., De Benedetti, M. (eds.) WOA 2016 - 17th Workshop "From Objects to Agents", CEUR-WS.org, vol. 1664, pp. 91-99. Sun SITE Central Europe (29-30 Jul 2016)

62. Mariani, S., Omicini, A., Sangiorgi, L.: Models of autonomy and coordination: Integrating subjective \& objective approaches in agent development frameworks. In: Braubach, L., Camacho, D., Venticinque, S., Bădică, C. (eds.) Intelligent Distributed Computing VIII, SCI, vol. 570, pp. 69-79. Springer International Publishing (2015)

63. Merrick, I., Wood, A.: Scoped coordination in open distributed systems. In: Porto and Roman [6], pp. 311-316

64. Minsky, N.H., Leichter, J.: Law-Governed Linda as a coordination model. In: Ciancarini, P., Nierstrasz, O., Yonezawa, A. (eds.) Object-Based Models and Languages for Concurrent Systems. LNCS, vol. 924, pp. 125-146. Springer (1994)

65. Mostinckx, S., Scholliers, C., Philips, E., Herzeel, C., De Meuter, W.: Fact Spaces: Coordination in the face of disconnection. In: Murphy and Vitek [67], pp. 268-285

66. Murphy, A.L., Picco, G.P., Roman, G.C.: LIME: A coordination model and middleware supporting mobility of hosts and agents. ACM Transactions on Software Engineering and Methodology (TOSEM) 15(3), 279-328 (Jul 2006)

67. Murphy, A.L., Vitek, J. (eds.): Coordination Models and Languages. 9th International Conference, COORDINATION 2007, Paphos, Cyprus, June 6-8, 2007. Proceedings, LNCS, vol. 4467. Springer (2007)

68. Ng, N., Yoshida, N., Pernet, O., Hu, R., Kryftis, Y.: Safe parallel programming with Session Java. In: De Meuter and Roman 32, pp. 110-126

69. Omicini, A.: Hybrid coordination models for handling information exchange among Internet agents. In: Bonarini, A., Colombetti, M., Lanzi, P.L. (eds.) Workshop "Agenti intelligenti e Internet: teorie, strumenti e applicazioni". pp. 1-4. 7th AI*IA Convention (AI*IA 2000), Milano, Italy (13 Sep 2000)

70. Omicini, A.: Formal ReSpecT in the A\&A perspective. Electronic Notes in Theoretical Computer Science 175(2), 97-117 (Jun 2007)

71. Omicini, A., Denti, E.: From tuple spaces to tuple centres. Science of Computer Programming 41(3), 277-294 (Nov 2001)

72. Omicini, A., Zambonelli, F., Klusch, M., Tolksdorf, R. (eds.): Coordination of Internet Agents: Models, Technologies, and Applications. Springer (Mar 2001)

73. Papadopoulos, G.A.: Models and technologies for the coordination of Internet agents: A survey. In: Omicini et al. 72], chap. 2, pp. 25-56

74. Picco, G.P., Murphy, A.L., Roman, G.C.: LIME: Linda meets mobility. In: 1999 International Conference on Software Engineering (ICSE 1999). pp. 368-377 (May 1999)

75. Plociniczak, H., Eisenbach, S.: JErlang: Erlang with Joins. In: Clarke, D., Agha, G. (eds.) Coordination Models and Languages, LNCS, vol. 6116, pp. 61-75. Springer (2010) 
76. Porto, A., Roman, G.C. (eds.): Coordination Languages and Models. 4th International Conference, COORDINATION 2000 Limassol, Cyprus, September 11-13, 2000 Proceedings, LNCS, vol. 1906. Springer (2000)

77. Proença, J., Clarke, D.: Interactive interaction constraints. In: De Nicola, R., Julien, C. (eds.) Coordination Models and Languages, LNCS, vol. 7890, pp. 211225. Springer (2013)

78. Proença, J., Clarke, D., de Vink, E., Arbab, F.: Dreams: A framework for distributed synchronous coordination. In: 27th Annual ACM Symposium on Applied Computing (SAC 2012). pp. 1510-1515. ACM, New York, NY, USA (2012)

79. Ricci, A., Omicini, A., Denti, E.: Virtual enterprises and workflow management as agent coordination issues. International Journal of Cooperative Information Systems 11(3/4), 355-379 (Sep/Dec 2002)

80. Rossi, D.: A social software-based coordination platform. In: Sirjani 90], pp. 17-28

81. Rossi, D., Cabri, G., Denti, E.: Tuple-based technologies for coordination. In: Omicini et al. 72], chap. 4, pp. 83-109

82. Rossi, D., Vitali, F.: Internet-based coordination environments and documentbased applications: a case study. In: Ciancarini and Wolf [26], pp. 259-274

83. Rowstron, A.I.T.: Bulk Primitives in Linda Run-Time Systems. Ph.D. thesis, The University of York (1996)

84. Rowstron, A.I.T.: Using asynchronous tuple-space access primitives (bonita primitives) for process co-ordination. In: Garlan and Le Métayer 43], pp. 426-429

85. Rowstron, A.I.T.: WCL: A co-ordination language for geographically distributed agents. World Wide Web 1(3), 167-179 (Sep 1998)

86. Sample, N., Beringer, D., Melloul, L., Wiederhold, G.: CLAM: Composition language for autonomous megamodules. In: Ciancarini and Wolf 26], pp. 291-306

87. Schumacher, M., Chantemargue, F., Hirsbrunner, B.: The STL ++ coordination language: A base for implementing distributed multi-agent applications. In: Ciancarini and Wolf [26, pp. 399-414

88. Sen, R., Roman, G.C., Gill, C.: CiAN: A workflow engine for MANETs. In: Lea and Zavattaro [56], pp. 280-295

89. Simperl, E., Krummenacher, R., Nixon, L.: A coordination model for triplespace computing. In: Murphy and Vitek 67], pp. 1-18

90. Sirjani, M. (ed.): Coordination Models and Languages. 14th International Conference, COORDINATION 2012, Stockholm, Sweden, June 14-15, 2012. Proceedings, LNCS, vol. 7274. Springer (2012)

91. Tarau, P.: Coordination and concurrency in multi-engine Prolog. In: De Meuter and Roman [32], pp. 157-171

92. Tolksdorf, R.: Coordinating services in open distributed systems with Laura. In: Ciancarini and Hankin [24], pp. 386-402

93. Tolksdorf, R.: Berlinda: An object-oriented platform for implementing coordination languages in Java. In: Garlan and Le Métayer [43], pp. 430-433

94. Tolksdorf, R., Rojec-Goldmann, G.: The SpaCETUB models and framework. In: Arbab and Talcott 7], pp. 348-363

95. Varela, C., Agha, G.: A hierarchical model for coordination of concurrent activities. In: Ciancarini and Wolf [26], pp. 166-182

96. Viroli, M., Omicini, A.: Coordination as a service. Fundamenta Informaticae 73(4), 507-534 (2006)

97. Zambonelli, F., Omicini, A., et al.: Developing pervasive multi-agent systems with nature-inspired coordination. Pervasive and Mobile Computing 17, 236-252 (Feb 2015) 